International Journal of Distributed and Parallel Systems (IJDPS) Vol.2, No.1, January 2011

\title{
Performance Evaluation of On Demand Routing Protocols AODV and Modified AODV (R-AODV) in MANETS
}

\author{
Humaira Nishat ${ }^{1}$, Vamsi Krishna $K^{2}$, Dr. D.Srinivasa Rao ${ }^{3}$ and Shakeel Ahmed ${ }^{4}$ \\ ${ }^{1}$ Department of Electronics and Communication Engineering, CVR College of \\ Engineering, Hyderabad, India. huma_nisha@yahoo.com \\ ${ }^{2}$ Department of Electronics and Communication Engineering, Jawaharlal Nehru \\ Technological University, Hyderabad, India. vamsikrishnagreat lgmail . com \\ ${ }^{3}$ Department of Electronics and Communication Engineering, Jawaharlal Nehru \\ Technological University, Hyderabad, India. dsraoecee jntuh. ac. in \\ ${ }^{4}$ Department of Electronics and Communication Engineering, Azad College of \\ Engineering and Technology, Hyderabad, India. shakeel_be@yahoo.com
}

\begin{abstract}
In mobile ad hoc networks, there is no centralized infrastructure to monitor or allocate the resources used by the mobile nodes. The absence of any central coordinator makes the routing a complex one compared to cellular networks. The Ad hoc On Demand Distance Vector (AODV) routing algorithm is a routing protocol designed for ad hoc mobile devices. AODV uses an on demand approach for finding routes. AODV and most of the on demand ad hoc routing protocols use single route reply along the reverse path. Due to rapid changes of topology the route reply may not arrive to the source node resulting in sending several route request messages and degrading the performance of the routing protocol. The extended AODV called Reverse Ad Hoc On Demand Vector (R-AODV) protocol uses a reverse route discovery mechanism and performs well when link breakage is frequent. In this paper we compare the QoS parameters such as Throughput, Delay and Packet Delivery ratio of both traditional $A O D V$ and R-AODV using TCP New Reno as the traffic source. Simulation results show that R-AODV performs well when link breakage is frequent.
\end{abstract}

\section{Keywords}

AODV, R-AODV, MANETs, QoS Parameter \& TCP New Reno

\section{INTRODUCTION}

Mobile ad hoc network [1], [2] is a dynamic network which allows communication between the mobile nodes without a central administrator. The network topology in such a network may keep changing randomly. Routing protocols used in traditional wired networks cannot be directly applied in ad hoc wireless networks due to their highly dynamic topology. A variety of routing protocols [7], [8] for ad hoc wireless networks have been proposed in the recent past.

Ad hoc wireless network routing protocols [7], [8] can be classified into three major categories based on the routing information update mechanism. 
International Journal of Distributed and Parallel Systems (IJDPS) Vol.2, No.1, January 2011

1. Proactive or table driven routing protocols: In this, each node maintains the network topology information in the form of routing tables by periodically exchanging routing information. Routing information is generally flooded in the whole network. Whenever a node needs a route to the destination it runs an appropriate path finding algorithm on the topology information it maintains.

2. Reactive or on demand routing protocols: Such protocols do not maintain the network topology information. They obtain the necessary route when it is required, by using a connection establishment process. Hence these protocols do not exchange routing information periodically.

3. Hybrid routing protocols: These protocols combine the best features of the above two categories. Nodes with a certain distance from the source node concerned or within a particular geographical region are said to be within the routing zone of the given node. For routing within this zone, a table-driven approach is used. For nodes located beyond this zone, an on-demand approach is used.

We focus our study on on-demand routing protocols. One of the on-demand routing protocol is AODV [4]. The main advantage of this protocol is that routes are established on demand i.e., only when it is required by a source node for transmitting data packets. But due to the dynamic change of network topology, links between nodes are not permanent. When a link breaks, a node cannot send packets to the intended next hop node resulting in packet loss. If the lost packet is a route reply packet it brings much more problems as the source node needs to reinitiate route discovery procedure.

In this paper we study the performance comparison of the modified AODV (R-AODV) [10] algorithm in which route reply message is multicast to its neighbors resulting in redundant route reply messages instead of unicasting the route reply to its next hop as in the traditional AODV. With this the probability of a successful route discovery is increased as we have repetitious route reply messages in our network. The robustness of the R-AODV algorithm is tested and compared with the existing AODV algorithm by using TCP New Reno as traffic source.

The route discovery procedure and design of AODV protocol is discussed by C. Pekin et al in [4]. The design of extended AODV(R-AODV) also called Reverse AODV and the comparative analysis of AODV with R-AODV using UDP traffic for constant bit rate applications considering scalability is discussed by E.Talipov et al in [10]. In this paper we compare the QoS parameters [7], [9] of both traditional AODV and R-AODV using TCP New Reno as the traffic source.

The rest of the paper is organized as follows: Section 2 gives a brief introduction of AODV routing protocol and an overview of modified AODV(R-AODV) routing protocol. Simulation setup is described in section 3. Section 4 gives the results and performance comparison of the two routing protocols. Section 5 concludes the paper.

\section{DESCRIPTION OF ROUTING PROTOCOLS}

\subsection{Ad hoc on demand distance vector (AODV)}

Ad hoc on demand distance vector (AODV) [3] routing protocol creates routes on-demand. In AODV, a route is created only when requested by a network connection and information regarding this route is stored only in the routing tables of those nodes that are present in the path of the route. 
International Journal of Distributed and Parallel Systems (IJDPS) Vol.2, No.1, January 2011

The procedure of route establishment is as follows. Assume that node $\mathrm{X}$ wants to set up a connection with node $\mathrm{Y}$. Node $\mathrm{X}$ initiates a path discovery process in an effort to establish a route to node $\mathrm{Y}$ by broadcasting a Route Request (RREQ) packet to its immediate neighbors. Each RREQ packet is identified through a combination of the transmitting node's IP address and a broadcast ID. The latter is used to identify different RREQ broadcasts by the same node and is incremented for each RREQ broadcast. Furthermore, each RREQ packet carries a sequence number which allows intermediate nodes to reply to route requests only with up-todate route information. Upon reception of an RREQ packet by a node, the information is forwarded to the immediate neighbors of the node and the procedure continues until the RREQ is received either by node $\mathrm{Y}$ or by a node that has recently established a route to node $\mathrm{Y}$. If subsequent copies of the same RREQ are received by a node, these are discarded.

When a node forwards a RREQ packet to its neighbors, it records in its routing table the address of the neighbor node where the first copy of the RREQ was received. This helps the nodes to establish a reverse path, which will be used to carry the response to the RREQ. AODV supports only the use of symmetric links. A timer starts running when the route is not used. If the timer exceeds the value of the 'lifetime', then the route entry is deleted.

Routes may change due to the movement of a node within the path of the route. In such a case, the upstream neighbor of this node generates a 'link failure notification message' which notifies about the deletion of the part of the route and forwards this to its upstream neighbor. The procedure continues until the source node is notified about the deletion of the route part caused by the movement of the node. Upon reception of the 'link failure notification message' the source node can initiate discovery of a route to the destination node.

\subsection{Modified AODV (R-AODV)}

Most of on-demand routing protocols, except multipath routing uses single route reply along the first reverse path to establish routing path. In high mobility, pre-decided reverse path can be disconnected and route reply message from destination to source can be missed. In this case, source node needs to retransmit route request message.

AODV protocol uses a single route reply message which may be lost in a network with mobile nodes. Transmission control protocols uses acknowledgements to confirm successful data transmission. When TCP is used as a transport layer protocol in MANET which employs AODV at network layer, it deteriorates the performance of the network when mobility is high.

The main purpose of our study is to increase the possibility of establishing routing path with less RREQ messages than the other protocol has, when topology changes by nodes mobility.

The modified AODV (R-AODV) [10] protocol discovers routes on-demand using a reverse route discovery procedure. During route discovery procedure source node and destination node plays some role from the point of sending control messages. Thus after receiving RREQ message, destination node floods reverse request (R-RREQ), to find source node. When source node receives an R-RREQ message, data packet transmission is started immediately. 
International Journal of Distributed and Parallel Systems (IJDPS) Vol.2, No.1, January 2011

\section{SIMULATION SETUP}

The R-AODV [8] protocol incorporates a route reply similar to route request in AODV [4]. To verify the hypothesis, we have implemented R-AODV by changing the source code of AODV in NS2 simulator [5], [6] to enable multiple route reply packets. The simulation setup is described in Table1.

Table 1. Simulation Parameters

\begin{tabular}{|l|l|}
\hline Parameter & Value \\
\hline Routing Protocols & AODV, R-AODV \\
\hline MAC Layer & 802.11 \\
\hline Terrain Size & $1315 \mathrm{~m} * 572 \mathrm{~m}$ \\
\hline No. of Nodes & 25 \\
\hline Mobility Model & Random Mobility Model \\
\hline Packet Size & $1500 \mathrm{~B}$ \\
\hline Bandwidth & $11 \mathrm{MB}$ \\
\hline Frequency & $2.472 \mathrm{GHz}$ \\
\hline Antenna Type & Omni antenna \\
\hline Propagation Model & $2-$ Ray ground \\
\hline Speed & $0-5-10-15-20-25 \mathrm{~m} / \mathrm{s}$ \\
\hline Simulation Time & $100 \mathrm{~s}$ \\
\hline Traffic Source & TCP New Reno \\
\hline Application Layer & FTP \\
\hline
\end{tabular}

We have built a validation module by constructing a scenario of 25 mobile nodes using TCL script. The awk script is run on the trace file obtained after the simulation in Linux Kernel to obtain the statistics of throughput, delay and packet delivery ratio. We make comparison between AODV and R-AODV under TCP New Reno considering the extracted statistics.

\subsection{Performance metrics}

Mobile ad hoc network routing protocols can be evaluated by a number of quantitative metrics described by RFC2501 [7]. We have used the following metrics for evaluating the performance of the two routing protocols (AODV \& R-AODV).

\subsubsection{Throughput}

It is the rate of successfully transmitted data packets per second in the network during the simulation. 
International Journal of Distributed and Parallel Systems (IJDPS) Vol.2, No.1, January 2011

\subsubsection{Average end-to-end delay}

It is defined as the average time taken by the data packets to propagate from source to destination across a MANET. This includes all possible delays caused by buffering during routing discovery latency, queuing at the interface queue, and retransmission delays at the MAC, propagation and transfer times.

\subsubsection{Packet Delivery Ratio}

It is the ratio of the number of packets received by the destination to the number of data packets generated by the source.

\section{SIMULATION RESULTS}

Here we present a comparative analysis of the performance metrics of both the on-demand routing protocols AODV and R-AODV with TCP New Reno as the traffic source for different node speeds $5,10,15,20 \& 25 \mathrm{~m} / \mathrm{s}$.

\subsection{Throughput}

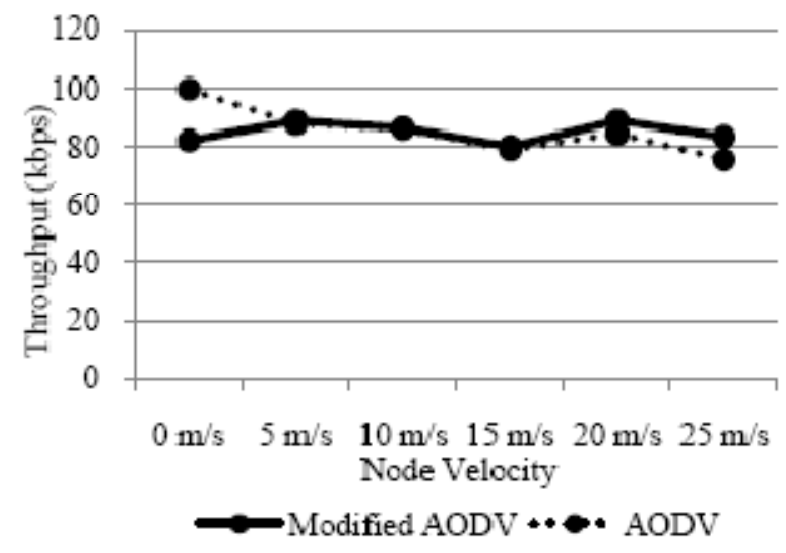

Fig. 1. Throughput, when mobile nodes velocity varies

From above figure it is clear that at $0 \mathrm{~m} / \mathrm{s}$, AODV gives better throughput than R-AODV. As the node mobility increases to $18 \mathrm{~m} / \mathrm{s}$ both AODV and R-AODV has almost same throughput but as the speed increases beyond 18m/s R-AODV outperforms AODV (as the throughput of AODV decreases with node velocity).

\subsection{Average Delay}

From figure 2 it is clear that AODV gives more delay than R-AODV routing protocol and it increases with the node velocity. Average delay is less for R-AODV routing protocol and is almost constant for various node velocity. Thus R-AODV gives better delay performance than AODV. 
International Journal of Distributed and Parallel Systems (IJDPS) Vol.2, No.1, January 2011

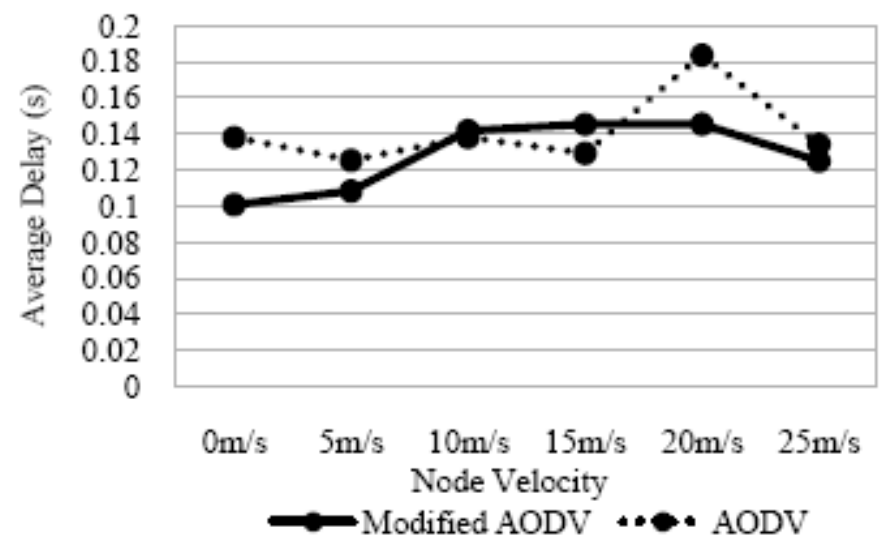

Fig. 2. Average delay, when mobile nodes velocity varies

\subsection{Packet Delivery Ratio}

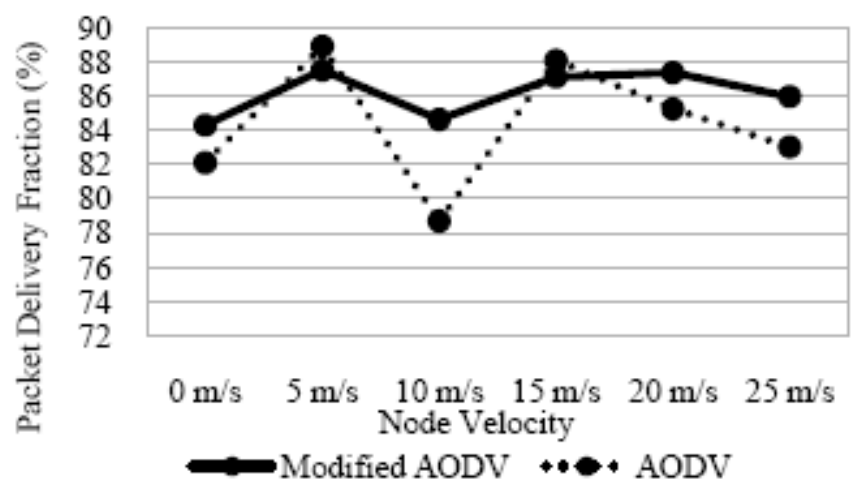

Fig. 3. Packet delivery ratio, when the mobile node velocity varies

Figure 3 gives the packet delivery ratio when the node velocity varies. With node velocity from 0 to $5 \mathrm{~m} / \mathrm{s}$ both AODV and R-AODV has almost same packet delivery ratio but as the velocity increases to $10 \mathrm{~m} / \mathrm{s}$ the ratio decreases rapidly in case of AODV whereas R-AODV maintains the same ratio. Thus with the increase in node velocity R-AODV gives more PDR thereby outperforming AODV.

\section{CONCLUSIONS}

Successful delivery of route reply message is very important in a MANET as a lot of route discovery effort is wasted if a reply message is lost, moreover a new route discovery process has to be reinitiated. Our simulation results show that R-AODV protocol has better throughput and average delay with increasing node velocity. Further multiple route reply messages in MANET results in a stable packet delivery ratio. Thus, with the increase in node velocity RAODV gives more PDR outperforming AODV. We validate the use of R-AODV with TCP New Reno traffic for FTP applications. 
International Journal of Distributed and Parallel Systems (IJDPS) Vol.2, No.1, January 2011

In this paper the two on-demand routing protocols AODV \& R-AODV are analyzed and their performances have been evaluated with respect to three performance metrics using TCP New Reno as the traffic source. This paper can be enhanced by analyzing other MANET routing protocols with different traffic sources.

\section{REFERENCES}

[1] M.S. Carson, S. Batsell and J. Macker, "Architecture consideration for Mobile Mesh Networking," Proceedings of the IEEE Military Communications Conference(MILCOM), vol.1, pp 225-229, 21-24 oct.1996.

[2] C.K. Toh “Ad Hoc Mobile Wireless Networks Protocols and Systems", First Edition, Prentice Hall Inc, USA 2002.

[3] N.Ch.sriman, Syed Mohammed Ansar, Sachin Kumar and Piyush Nagas "An Efficient and Secure Routing Protocol for Mobile Ad-hoc Networks", IJCNC, Volume 2, No.3, May 2010 .

[4] C.Perkin, Elizabeth M. Royer, "Ad hoc on demand Distance Vector Routing”, RFC 3561, July 2003, http.//www.ietf.org/rfc/rfc3561.txt.

[5]UCB/LBNL/VINT Network Simulator, http://wwwmash.cs.berkeley.edu/ns/referred on March 2010.

[6] “The Network Simulator- ns-2", available at http://www.isi.edu/nsnam/ns/referred on march 2010.

[7] S.Corson and J.Macker, "Routing Protocol performance Issues and Evalaution Considerations", RFC2501, IETF Network Working Group, January 1999.

[8]N.H.Vaidya, "Mobile Ad Hoc Networks Routing, MAC and transport Issues", Proceedings of the IEEE International Conference on Computer Communication INFOCOM, 2004.

[9] L.Layuan, Y.Peiyan and L.Chunlin, "Performance Evaluation and Simulations of Routing Protocols in Ad Hoc Networks” Computer Communications, vol.30, pp.1890-1998, 2007.

[10] C Kim, E.Talipov, and B.Ahn, A Reverse AODV (R-AODV) Routing Protocol in ad hoc Mobile Networks, in the 2006 IFIP International Conference on Embedded and Ubiquitous Computing" (EUC'06), LNCS 4097, Seoul, Korea, August 2006, pp.522-531.

[11] Mehdi Zarei, Karim Faez and Javad Moosavi Nya, "Modified Reverse AODV Routing Algorithm using

Route Stability in Mobile Ad Hoc Networks" Proceedings of the 12th IEEE International Multi topic Conference, December 23-24, 2008.

[12] Zhi Li and Yu-Kwong Kwok, "A New Multipath Routing Approach to Enhancing TCP Security in Ad Hoc Wireless Networks" in Proc. ICPPW 2005.

[13] Chonggun Kim, Elmurod Talipov, and Byoungchul Ahn, "A Reverse AODV Routing Protocol in Ad Hoc Mobile Networks", LNCS 4097, pp. 522 531,2006.

[14] Tarng, B.Chuang and F. Wu , "Link Stability-based Routing Protocol for Mobile Ad Hoc Networks", 2006 IEEE Conference on Systems, Man, and Cybernetics October 8-11, 2006, Taipei, Taiwan.

[15] L. Layuan, L. Chunlin, Y. Peiyan, "Performance evaluation and simulations of routing protocols in ad hoc networks", s.l. : Elsevier, Computer Communications, 2007.

[16] H. Pucha, S. M. Das, Y. C. Hu. The performance impact oftraffic patterns on routing protocols in 
International Journal of Distributed and Parallel Systems (IJDPS) Vol.2, No.1, January 2011

mobile ad hoc networks. s.l. : Elsevier, Computer Networks, 2007.

[17] Lu, Y., Weichao, W., Zhong, Y., Bhargava, B.: "Study of Distance Vector Routing Protocols for Mobile ad hoc networks"; Proceedings of the First IEEE International Conference 2003.

[18] Min Sheng, jiangdong Li and Van Shi, "Delay Sensitive Adaptive Routing Protocol for Ad Hoc Network," 17 th International Conference on Advanced Information Networking and Applications (AINAt03), March 27-29,2003.

[19] Izhak Rubin and Y. C. Liu, "Link Stability Models for QoS Ad Hoc Routing Algorithms," Vehicular Technology Conference, 2003. VTC 2003-Fall. 2003 IEEE 58th.

[20] Chandra Kant Samal, "TCP Performance through Simulation and Testbed in Multi-hop Mobile Ad hoc Network", IJCNC Volume.2, No.4, July 2010.

\section{Authors}

1.Humaira Nishat received the B-Tech degree in Electronics and Communication Engineering in the year 2000 and M-Tech degree in Digital Systems \& Computer Electronics in the year 2004 from Jawaharlal Nehru Technological University Hyderabad, India. She is currently working towards the $\mathrm{PhD}$ degree in the department of Electronics and Communication Engg, Jawaharlal Nehru Technological University Hyderabad, India. Her research interests include Wireless Communications and Networks.

2.Vamsi K. Konakalla received his B-Tech degree in Electronics and Communication Engineering from SASTRA University, India in 2009. He is currently pursuing his M.Sc. at Blekinge Institute of Technology, Karlsrona, Sweden as an exchange student from Jawaharlal Nehru Technological University (JNTU), Hyderabad, India.His current research interests are Quality of service improvisation in Mobile Ad-Hoc networks and network measurements.

3.Dr. D.Srinivasa Rao, received the B-Tech degree in Electronics and Communication Engineering from Nagarjuna University, India in 1986 and M.E degree in Digital Systems, Osmania University, India in 1994, Phd degree in Computer Networks from University of Hyderabad, India in 2004. He is currently working as Professor in the dept. of ECE, Jawaharlal Nehru Technological University Hyderabad, India.

4 Shakeel Ahmed received the B-Tech degree in Electronics and Communication Engineering from Bangalore University, India in 1997 and M.Tech degree in Digital Communications \& Networking from Vinayaka Mission University, India in 2007. His research interests include Wireless \& Computer Networks. He is currently working as Associate Professor in Dept of ECE, Azad college of Engineering and Technology, Hyderabad, India. He is a member of IETE.
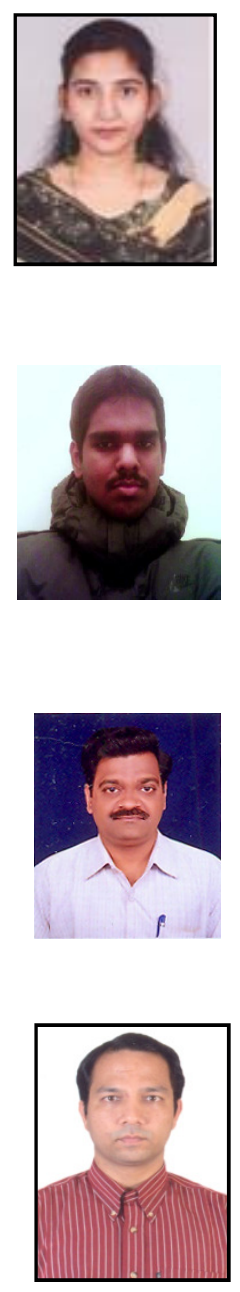